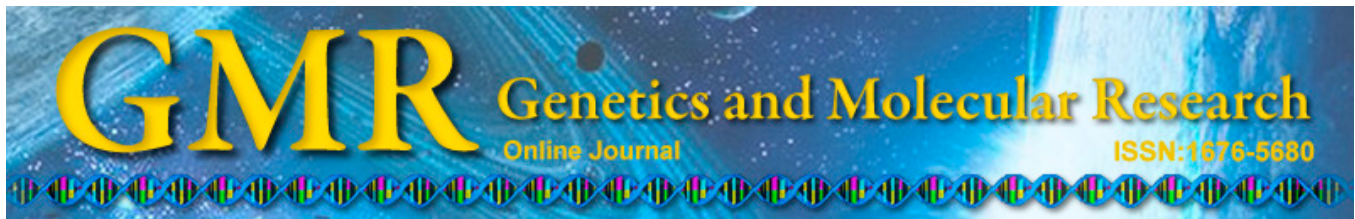

\title{
Values for serum procalcitonin, $C$-reactive protein, and soluble triggering receptor expressed on myeloid cells- 1 in predicting survival of patients with early-onset stroke-associated pneumonia
}

\author{
J. Xie', X.H. Zhang' and W.Y. Zhu' \\ ${ }^{1}$ Department of Geriatrics, The Fifth People's Hospital of Shanghai, \\ Fudan University, Shanghai, China \\ ${ }^{2}$ Department of Emergency Medicine, The Fifth People's Hospital of Shanghai, \\ Fudan University, Shanghai, China \\ Corresponding author: J. Xie \\ E-mail: xiejuansci@163.com
}

Genet. Mol. Res. 14 (2): 4716-4723 (2015)

Received June 17, 2014

Accepted October 20, 2014

Published May 11, 2015

DOI http://dx.doi.org/10.4238/2015.May.11.4

\begin{abstract}
We examined the value of serum procalcitonin (PCT), $\mathrm{C}$-reactive protein (CRP), soluble triggering receptor expressed on myeloid cells-1 (sTREM-1) for predicting the survival of patients with early-onset stroke associated pneumonia (EOP). A total of 207 stroke patients were enrolled, and 91 developed EOP. Upon admission, serum PCT, CRP, sTREM-1 levels, clinical pulmonary infection score, and Acute Physiology and Chronic Health Evaluation II score were all significantly higher in patients with EOP than in those without EOP $(\mathrm{P}<0.05)$. Of the 91 patients who developed EOP, 39 (42.9\%) died (non-survivors) within 28 days. The Acute Physiology and Chronic Health Evaluation II score on admission was significantly higher in non-survivors than in survivors $(\mathrm{P}<0.05)$.
\end{abstract}


Serum PCT and sTREM-1 levels were slightly elevated on days 1,3 , and 5 in non-survivors and gradually decreased in survivors. Serum PCT, sTREM-1, and CRP levels were all significantly higher in non-survivors than in survivors on days 1,3 , and $5(\mathrm{P}<0.05)$. The sensitivity and specificity of PCT for predicting the outcome of EOP were 84.6 and $71.2 \%$, the sensitivity and specificity of sTREM- 1 were 71.8 and $92.3 \%$, and the sensitivity and specificity of sTREM-1 combined with PCT were 74.4 and $96.2 \%$. Serum PCT combined with sTREM-1 accurately predicted the outcome of EOP patients, and dynamic monitoring of serum PCT and sTREM-1 levels is necessary.

Key words: Clinical pulmonary infection score; C-reactive protein; Early-onset stroke-associated pneumonia; Procalcitonin; Prognosis;

Soluble triggering receptor expressed on myeloid cells-1

\section{INTRODUCTION}

Pneumonia is one of the most common complications of acute stroke, and generally results in aggravation and death. Hilker et al. (2003) defined this type of pneumonia as stroke-associated pneumonia (SAP), which differs from nosocomial pneumonia in stroke patients (Myrianthefs et al., 2004). Early-onset stroke associated pneumonia (EOP) is SAP that occurs within $72 \mathrm{~h}$ after stroke onset, contributing to $73 \%$ of reported SAP cases (Hilker et al., 2003). EOP is associated with increased in hospital mortality, poor discharge outcome, increased period of hospitalization, and increased medical costs. Therefore, it is important to identify indicators for adequately determining the condition of EOP patients, particularly for patients with poor outcomes. Recently, procalcitonin (PCT), soluble triggering receptor expressed on myeloid cells-1 (sTREM-1), and C-reactive protein (CRP) have been reported as inflammation markers of sepsis in ventilator-associated pneumonia (VAP) patients (Ventetuolo and Levy, 2008; Hillas et al., 2010; Su et al., 2012), however, there are very few reports that suggest the use of these indicators to predict the outcome of EOP patients. In this study, serum PCT, CRP, and sTREM-1 levels in EOP patients were dynamically monitored on days 1,3 , and 5 , and the value of these indicators in predicting the outcome of EOP patients was examined.

\section{MATERIAL AND METHODS}

\section{Patients}

From June 2011 to January 2013, 232 stroke patients were admitted to the emergency intensive care unit (EICU), the Fifth People's Hospital of Shanghai, including 128 males and 104 females, aged from 19-90 years (mean age, $64.6 \pm 9.6$ years). Acute stroke was diagnosed clinically according to the cerebrovascular disease diagnostic criteria issued at the Fourth National Cerebrovascular Diseases Conference in China, and all confirmed 
by computed tomography $(\mathrm{CT})$ and magnetic resonance imaging examination. All patients were admitted to the hospital within $72 \mathrm{~h}$ after stroke onset. The diagnostic criteria for SAP were as follows (more than 2 items coexisted): 1) body temperature $>38^{\circ} \mathrm{C}, 2$ ) purulent respiratory secretions, 3$)$ newly diagnosed hypoxemia $\left(\mathrm{PaO}_{2}<70\right.$ or $10 \mathrm{mmHg}$ less than the basic oxygen partial pressure), 4) bacterial pathogens found in smear or sputum culture, 5) white blood cell count $>10.0 \times 10^{9} / \mathrm{L}$, and 6) new effusion lesions on imaging (chest CT or X-ray). EOP is defined as SAP developed with $72 \mathrm{~h}$ after stroke onset. Exclusion criteria were as follows: 1) discharge or death within $24 \mathrm{~h}, 2$ ) brain death or heartbeat stop on admission, 3) positive results of tracheal secretions, sputum smear, or culture, or pneumonia confirmed by chest X-ray. Among all 232 patients, 13 died within $24 \mathrm{~h}$ of admission, pneumonia was confirmed in 12 cases on admission, and a total of 207 patients were enrolled in the study. Antibiotics were administered to all patients within $72 \mathrm{~h}$ in the EICU. All patients were subgrouped into the EOP group or the non-EOP group. This study was approved by the Ethics Committee of the Fifth People's Hospital of Shanghai, Fudan University, and written informed consent was obtained from all participants.

\section{Clinical data and examination}

Patient age, gender, and Glasgow Coma Scale score on admission were recorded, and the period of EICU stay and outcome (death or hospital discharge as the end point) were also recorded. For all study subjects, white blood cell counts, temperature, blood gas analysis, X-ray examination, tracheal secretions culture, clinical pulmonary infection score (CPIS) evaluation [with use of the simplified CPIS scale issued by Upadya et al. ( 2004)], Acute Physiology and Chronic Health Evaluation II (APACHE II) score evaluation and serum PCT, sTREM-1, and CRP levels were evaluated upon admission. Serum PCT, sTREM-1, and CRP levels were again assessed on days 3 and 5. PCT was assessed using a solid phase semi-quantitative immunoassay method, CRP was assessed using the turbidity immunization rate scattering method, and sTREM-1 was assessed using solid phase enzyme-linked immunosorbent assay. All kits were purchased from R\&D Systems, Inc. (Minneapolis, MN, USA). Blood samples were extracted and sent to the laboratory within $0.5 \mathrm{~h}$.

Patients developed EOP were followed for up to 28 days, and subgrouped into the survival group and non-survival group according to the outcome.

\section{Statistical analysis}

Normally distributed data are reported as means \pm standard deviation. Comparison among multiple sets of data was performed using analysis of variance for equal variance or Kruskal-Wallis $\mathrm{H}$ test for unequal variances. Non-normally distributed data were expressed as the median with interquartile (25-75\%) range, and Kruskal-Wallis H test was applied to compare multiple sets. Receiver operating characteristic curves were constructed to determine the optimal cut-off values, sensitivity, specificity, and area under curve (AUC). A P value $<0.05$ was considered to be statistically significant. 


\section{RESULTS}

\section{General condition}

Of all 207 stroke patients, EOP developed in 91 cases. Age, gender, and Glasgow Coma Scale score on admission of EOP patients were not significantly different $(\mathrm{P}>0.05)$ from those of patients who did not develop EOP. The period of EICU stay and mortality rate were significantly higher in patients with EOP $(\mathrm{P}<0.05)$. On admission, serum PCT, CRP, and sTREM-1 levels, CPIS score, and APACHE II score of patients who developed EOP were comparable to patients who did not develop EOP $(\mathrm{P}<0.05)$. The results are shown in Table 1.

Table 1. Demographic and clinical data of patients upon admission and outcomes.

\begin{tabular}{lccc}
\hline Characteristics & Non-EOP & EOP & P value \\
\hline Patients (N) & 116 & 91 & - \\
Gender (M/F) & $64 / 52$ & $53 / 38$ & 0.658 \\
Age (years) & $65.1 \pm 9.8$ & $63.2 \pm 9.2$ & 0.157 \\
GCS & $6.0 \pm 1.7$ & $5.7 \pm 1.6$ & 0.198 \\
APACHE II score & $10.3 \pm 2.8$ & $17.6 \pm 4.2$ & $<0.05$ \\
EICU stay (days) & $6.7 \pm 2.9$ & $17.4 \pm 6.3$ & $<0.05$ \\
28-day mortality rate (N, \%) & $18(15.5 \%)$ & $39(42.9 \%)$ & $<0.05$ \\
PCT $(\mu \mathrm{L} / \mathrm{L}$ ) & $0.22(0.16-0.41)$ & $1.15(0.8-1.68)$ & $<0.05$ \\
sTREM-1 (ng/L) & $10.5(8-15.8)$ & $130(117-157)$ & $<0.05$ \\
CRP $(\mathrm{mg} / \mathrm{dL})$ & $1.2(0.9-1.6)$ & $10.6(7.7-15.4)$ & $<0.05$ \\
CPIS score & $1(1-2)$ & $3(2-4)$ & $<0.05$ \\
\hline
\end{tabular}

\section{Serum PCT, CRP, and STREM-1 levels of EOP patients on days 1, 3, and 5}

Among the 91 EOP patients, 39 died (non-survivors) within 28 days, with a mortality rate of $42.9 \%$. The APACHE II score of non-survivors on day 1 was significantly higher than that of survivors $(20.4 \pm 5.4$ vs $15.9 \pm 3.9, \mathrm{t}=4.62, \mathrm{P}<0.05)$. Serum PCT, CRP, and sTREM-1 levels of patients on days 1, 3, and 5 are shown in Table 2 and Figure 1. Serum levels of PCT and sTREM-1 of non-survivors were slightly elevated, while those of survivors were slightly decreased, and the serum levels of PCT, sTREM-1 and CRP at different time points were all significantly higher in non-survivors than in survivors $(\mathrm{P}<$ $0.05)$.

Table 2. Dynamic monitoring of inflammation markers in patients with EOP with different outcomes.

\begin{tabular}{lccccc}
\hline Outcome & Time & PCT $(\mu \mathrm{g} / \mathrm{L})$ & sTREM-1 $(\mathrm{ng} / \mathrm{L})$ & CRP $(\mathrm{mg} / \mathrm{dL})$ & APACHE II score \\
\hline Survived $(\mathrm{N}=52)$ & D1 & $0.91(0.75-1.38)$ & $121(109.5-130.75)$ & $9.35(6.58-12.3)$ & $15.9 \pm 3.9$ \\
& D3 & $0.73(0.62-1.03)$ & $90(75-108.5)$ & $8.5(6-10.5)$ & - \\
\multirow{2}{*}{ Died (N=39) } & D5 & $0.51(0.39-0.78)$ & $71(55-88.5)$ & $6.95(5.2-9.13)$ & - \\
& D1 & $1.68(1.38-4.13)$ & $164(135-206)$ & $13.4(10.5-18.2)$ & $20.4 \pm 5.4$ \\
& D3 & $2.89(1.27-5.43)$ & $182(154-223)$ & $11.8(9.4-16.2)$ & - \\
\hline
\end{tabular}



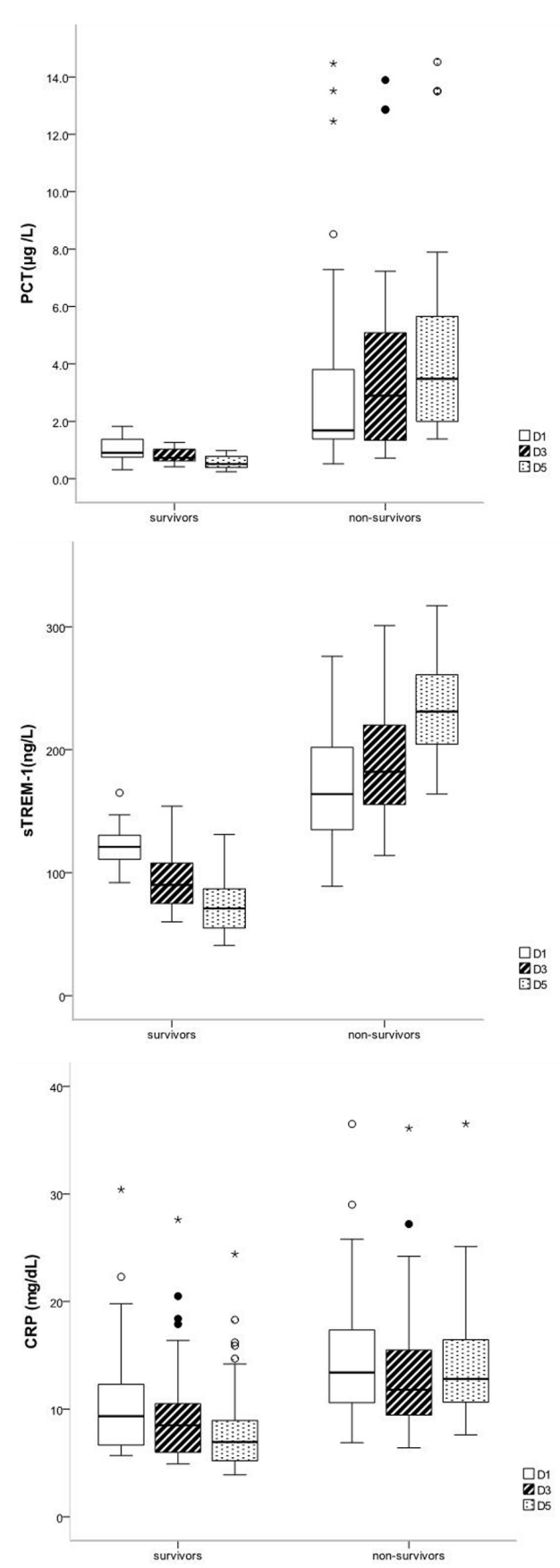

Figure 1. Dynamic monitoring of serum PCT (top), sTREM-1(middle), and CRP (bottom) in survivors and nonsurvivors. Box plots represent the 25th and 75th percentiles; the internal horizontal line shows the median and whiskers show the 10th and 90th percentiles. Stars and circles represent outliers. Comparison between groups, $\mathrm{P}<$ 0.05 on days 1,3 , and 5 for PCT, sTREM-1, and CRP. 


\section{Serum PCT, sTREM-1, CRP levels, and APACHE II score for predicting the sur- vival of EOP}

Serum PCT, sTREM-1, CRP levels and APACHE II score upon admission were used to predict patient outcome, and the results are shown in Table 3. For sTREM-1 and PCT, the AUCs were 0.871 and 0.854 , respectively. The sensitivity and specificity of PCT $>1.21 \mu \mathrm{g} / \mathrm{L}$ combined with sTREM-1 $>147 \mathrm{ng} / \mathrm{L}$ to predict death of EOP patients were 74.4 and $96.2 \%$, with an AUC of 0.893 (Figure 2).

Table 3. Serum PCT, sTREM-1, and CRP levels and APACHE II score upon admission for predicting patient
outcome.
\begin{tabular}{lcccc}
\hline Indicators & Cut off value & AUC $(95 \% \mathrm{CI})$ & Sensitivity $(\%)$ & Specificity $(\%)$ \\
\hline PCT $(\mu \mathrm{g} / \mathrm{L})$ & 1.23 & $0.854(0.772-0.936)$ & 84.6 & 71.2 \\
sTREM-1 $(\mathrm{ng} / \mathrm{L})$ & 138 & $0.871(0.791-0.950)$ & 71.8 & 92.3 \\
CRP $(\mathrm{mg} / \mathrm{dL})$ & 10.4 & $0.762(0.665-0.860)$ & 82.1 & 67.3 \\
APACHE II score & 19.4 & $0.738(0.633-0.842)$ & 53.8 & 84.6 \\
\hline
\end{tabular}

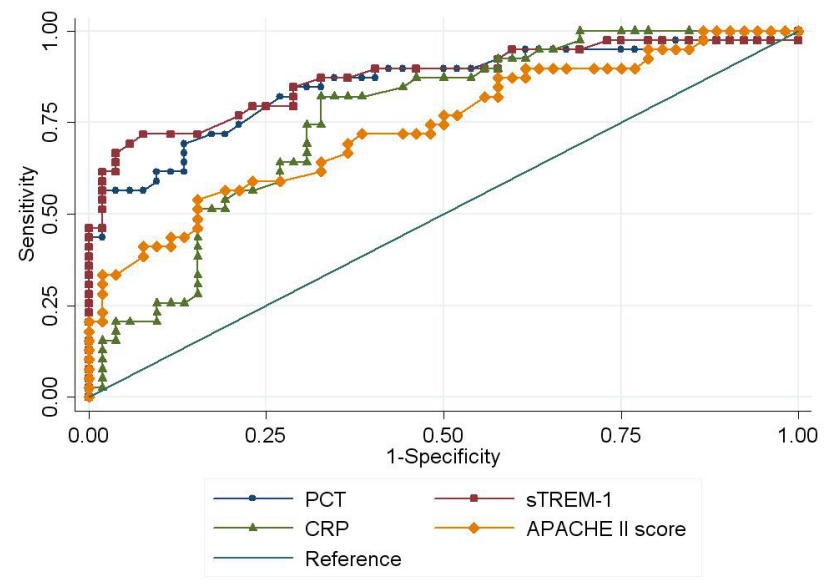

Figure 2. ROC curves of serum PCT, sTREM-1, and CRP levels and APACHE II score for predicting the outcome of patients with EOP.

\section{DISCUSSION}

The mortality rate of SAP in severe stroke in neonatal intensive care unit was $47.3 \%$, as reported (Upadya et al., 2004), while the mortality rate of EOP was 44\% in this study, which is slightly lower. Accurately diagnosing EOP is difficult because of the many restrictions of the patients studied, such as coma, low reactivity, less cough, difficulty in finding lesions on bedside chest radiograph, among other reasons (Emsley and Hopkins, 2008). In this study, serum PCT, CRP, and sTREM-1 levels and CPIS upon admission of EOP patients were compared with those of non-EOP patients. The results showed that the inflammation markers were significantly elevated in EOP patients and, thus, can be used for the early diagnosis of EOP.

Through dynamic monitoring of inflammation markers of EOP patients, we observed 
that serum PCT and sTREM-1 levels gradually increased in non-survivors and gradually decreased in survivors. PCT is widely applied for monitoring the state of illness of systemic inflammation (Wacker et al., 2013), and serum PCT level has been used to indicate the severity of systemic inflammation and to predict survival (Seligman et al., 2006). Hillas et al. (2010) reported that the dynamic change in PCT level is related to the outcome of ventilatorassociated pneumonia patients. sTREM-1, a biological marker of the inflammatory response, is important in the secretion of pro-inflammatory mediators and for suppressing the production of anti-inflammatory factors, and the pathway mediated that plays a key role in the development and amplification of the inflammatory response (Bouchon et al., 2001). Serum PCT and sTREM-1 levels gradually increased in non-survivors, indicating that the systemic inflammatory response was continually initiated and amplified. This led to the continued release of inflammatory factors and pro-inflammatory mediators, resulting in an imbalance between the pro- and anti-inflammatory response, worsening the condition of patients and increasing the chance of poor outcome. In contrast, the condition of patients improved when the serum PCT and sTREM-1 level gradually decreased. In agreement with previous research results ( $\mathrm{Su}$ et al., 2013), we also revealed that CRP level did not increase during the more serious stage of inflammation in non-survivors, indicating that CRP level is not related to infection severity and cannot be used to predict patient outcome.

The clinical features of EOP are atypical and cannot be applied for predicting outcome. The APACHE II scoring system is now widely applied for evaluating the severity of illness and the prognosis of ICU patients. However, the score is somewhat related to the management in ICU because the worst score within $24 \mathrm{~h}$ rather than the score upon admission is required (Ho et al., 2006), and it is not stable for evaluating the severity of illness and the prognosis of neonatal intensive care patients (Kim and Yoon, 2012). Therefore, its application in this study has some limitations. In contrast, inflammatory markers such as serum PCT, sTREM-1, and CRP may be ideal predictors because they can be monitored during early stages of management (Gibot et al., 2004), particularly sTREM-1, which can be monitored during early stages, with a short half-life and the level of that directly reflects the infection. This study revealed that the performance of sTREM-1 and PCT is better for predicting the survival of EOP patients than CRP and APACHE II score. PCT showed high sensitivity and sTREM-1 showed high specificity. The combination of the 2 indicators can be used to improve outcome prediction, with a specificity of $96.2 \%$ and AUC of 0.893 . Therefore, simultaneously monitoring of sTREM-1 and PCT is necessary for EOP patients.

There were some limitations to this study. Patients with positive results in microbial culture were excluded; therefore, the study results cannot be applied for patients with pulmonary infection upon admission. In addition, prophylactic treatment with antibiotics affects serum PCT level and pneumonia development. The sample number was limited, and more accurate results may be achieved in prospective clinical trials with larger samples.

In this study, serum PCT, sTREM-1, and CRP levels were first applied for predicting the survival of EOP patients. The results showed that dynamic monitoring of serum inflammation makers is necessary for EOP patients to help predict patient outcome, and thus for selecting active therapeutic strategies and reducing mortality.

\section{ACKNOWLEDGMENTS}

Research supported by the Key Discipline Construction Project of Shanghai Public 
Health-Emergency Medicine (Project \#08GWD05). We also thank Dr. Qiao Bin for critically reading this manuscript.

\section{Conflicts of interest}

The author declare no conflict of interest.

\section{REFERENCES}

Bouchon A, Facchetti F, Weigand MA and Colonna M (2001). TREM-1 amplifies inflammation and is a crucial mediator of septic shock. Nature 410: 1103-1107.

Emsley HC and Hopkins SJ (2008). Acute ischaemic stroke and infection: recent and emerging concepts. Lancet Neurol. 7: 341-353.

Gibot S, Kolopp-Sarda MN, Bene MC, Cravoisy A, et al. (2004). Plasma level of a triggering receptor expressed on myeloid cells-1: its diagnostic accuracy in patients with suspected sepsis. Ann. Intern. Med. 141: 9-15.

Hilker R, Poetter C, Findeisen N, Sobesky J, et al. (2003). Nosocomial pneumonia after acute stroke: implications for neurological intensive care medicine. Stroke 34: 975-981.

Hillas G, Vassilakopoulos T, Plantza P, Rasidakis A, et al. (2010). C-reactive protein and procalcitonin as predictors of survival and septic shock in ventilator-associated pneumonia. Eur. Respir. J. 35: 805-811.

Ho KM, Dobb GJ, Knuiman M, Finn J, et al. (2006). A comparison of admission and worst 24-hour Acute Physiology and Chronic Health Evaluation II scores in predicting hospital mortality: a retrospective cohort study. Crit. Care 10: R4.

Kim TK and Yoon JR (2012). Comparison of the predictive power of the LODS and APACHE II scoring systems in a neurological intensive care unit. J. Int. Med. Res. 40: 777-786.

Myrianthefs PM, Kalafati M, Samara I and Baltopoulos GJ (2004). Nosocomial pneumonia. Crit. Care Nurs. Q 27: 241257.

Seligman R, Meisner M, Lisboa TC, Hertz FT, et al. (2006). Decreases in procalcitonin and C-reactive protein are strong predictors of survival in ventilator-associated pneumonia. Crit. Care 10: R125.

Su L, Han B, Liu C, Liang L, et al. (2012). Value of soluble TREM-1, procalcitonin, and C-reactive protein serum levels as biomarkers for detecting bacteremia among sepsis patients with new fever in intensive care units: a prospective cohort study. BMC Infect. Dis. 12: 157.

Su L, Feng L, Song Q, Kang H, et al. (2013). Diagnostic value of dynamics serum sCD163, sTREM-1, PCT, and CRP in differentiating sepsis, severity assessment, and prognostic prediction. Mediators Inflamm. 2013: 969875.

Upadya A, Thorevska N, Sena KN, Manthous C, et al. (2004). Predictors and consequences of pneumonia in critically ill patients with stroke. J. Crit. Care 19: 16-22.

Ventetuolo CE and Levy MM (2008). Biomarkers: diagnosis and risk assessment in sepsis. Clin. Chest. Med. 29: 591-603.

Wacker C, Prkno A, Brunkhorst FM and Schlattmann P (2013). Procalcitonin as a diagnostic marker for sepsis: a systematic review and meta-analysis. Lancet Infect. Dis. 13: 426-435. 